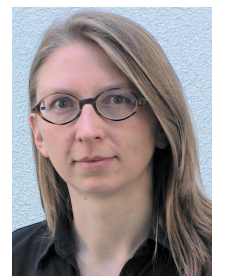

H. Kaduszkiewicz

\title{
Die hausärztliche Versorgung von Patienten mit kognitiven Störungen und Demenz
}

Hanna Kaduszkiewicz, Hendrik van den Bussche

Institut für Allgemeinmedizin, Universitätsklinikum Hamburg-Eppendorf

psychoneuro 2003; 29 (6): 275-281

„Der Arbeitsbereich der Allgemeinmedizin beinhaltet die Grundversorgung aller Patienten mit körperlichen und seelischen Gesundheitsstörungen (..). Allgemeinärztinnen und Allgemeinärzte sind darauf spezialisiert, als erste ärztliche Ansprechpartner bei allen Gesundheitsproblemen zu helfen. (..) Das Arbeitsziel der Allgemeinmedizin ist eine qualitativ hochstehende Versorgung, die den Schutz des Patienten, aber auch der Gesellschaft vor Fehl-, Unter- oder Überversorgung einschließt.“

$\mathrm{D}$ ieses Zitat entstammt der im September 2002 beschlossenen Fachdefinition der Deutschen Gesellschaft für Allgemeinmedizin und Familienmedizin (DEGAM). Im bundesrepublikanischen Alltag spielt der Hausarzt bei der Versorgung von Patienten mit kognitiven Störungen eine entscheidende Rolle. Die Versorgung von Heimpatienten liegt praktisch vollständig in hausärztlicher Hand. Wie aber sieht - gemessen an der DEGAM-Zielformulierung - die Wirklichkeit der Versorgung aus? Welche Defizite sind erkennbar?

Zunächst werden in diesem Beitrag die wenigen verfügbaren Studien zur hausärztlichen Versorgung

Wie sieht die Wirklichkeit der hausärztlichen Versorgung von Patienten mit kognitiven Störungen und Demenz aus? Diesbezüglich werden in diesem Beitrag die verfügbaren Studien untersucht. Ob Hausärzte kognitive Defizite übersehen und die Diagnose einer Demenz zu selten stellen, ist aufgrund eines fehlenden Standards in Bezug auf die Diagnosestellung schwierig festzumachen. Deutlich werden hingegen Hindernisse auf verschiedenen Ebenen, die die Frühdiagnose einer Demenz erschweren. Auf dem Hintergrund der wissenschaftlichen Literatur wird nachfolgend dargestellt, welchen Beitrag das hausärztliche Versorgungsprojekt des Kompetenznetzes Demenzen zur weiteren Klärung problematischer Bereiche der hausärztlichen Versorgung leisten will.

von Demenzpatienten untersucht Berücksichtigt wurden wissenschaftliche Veröffentlichungen der Jahre 1989 bis 2002, die unter den Schlagwörtern „family-practice bzw. general-practice bzw. primaryhealth-care“ und „dementia“ in den Datenbanken Medline und Embase verzeichnet waren. Auf diesem Hintergrund wird nachfolgend dargestellt, welchen Beitrag das hausärztliche Versorgungsprojekt des Kompetenznetzes Demenzen zur weiteren Klärung dieser Problematik leisten will.

\section{Die Erkennung kognitiver \\ Defizite durch Hausärzte}

Eine Vielzahl von Studien zu Demenz und hausärztlicher Versorgung beschäftigt sich mit dem Problem der Erkennung kognitiver Defizite durch den Hausarzt ohne Zuhil- fenahme von neuropsychologischen Testverfahren. Die Ergebnisse sind nicht einheitlich. In mehreren Studien wird den Hausärzten eine mangelnde Sensitivität in Bezug auf die Erkennung bescheinigt.

So wurden die Hausärzte in einer niederländischen Studie (6) gebeten, bei allen ihren gut bekannten über 65-jährigen Patienten, die nicht in einem Pflegeheim lebten, den kognitiven Status einzuschätzen. Bei 2,2\% dieser Patienten diagnostizierten die Hausärzte eine Demenz anhand der DSM-III-R Kriterien. Im Vergleich dazu diagnostizierten die Studienärzte unter Zuhilfenahme des CAMDEX bei 5,2\% der Studienpopulation eine Demenz.

Studien, die ein zu seltenes Erkennen kognitiver Defizite in der hausärztlichen Versorgung postulieren, leiden allerdings an methodi- 
schen Mängeln. So erfolgte in der oben zitierten Studie die Einschätzung der Hausärzte auf Basis ihrer Erinnerung. Der Einschätzung durch die Studienärzte ging hingegen ein direkter Patientenkontakt voraus, was deren Treffsicherheit erhöht haben dürfte. In anderen Studien, in den eine mangelnde Sensitivität von Hausärzten in Bezug auf die Erkennung kognitiver Störungen festgestellt wird, (z.B. 9) taucht häufig ein anderes Problem auf: Die Einschätzung der kognitiven Leistungsfähigkeit durch die Hausärzte wird mit einem Screeningtest und nicht mit einem diagnostischen Test verglichen. Da Screeningtests im Niedrig-Prävalenz-Bereich der Hausarztpraxis aber viele falsch positive Ergebnisse liefern, kann man nicht mit Sicherheit sagen, wie viele Patienten mit kognitiven Störungen von den Hausärzten tatsächlich übersehen wurden.

In einer methodisch sehr guten Studie (4) in 24 Mannheimer Praxen wurde im Gegensatz zu den oben zitierten Studien eine gute Fähigkeit der Hausärzte gefunden, Demenzerkrankungen - auch solche leichteren Grades - zu erkennen: Die Sensitivität betrug $92 \%$, die Spezifität $76 \%$. Zusammenfassend muss man also sagen, dass die Frage ob - und wenn $\mathrm{ja}$, in welchem Maße - Hausärzte kognitive Störungen bei ihren Patienten übersehen, nicht abschließend geklärt ist.

\section{Unklare Diagnosekriterien in der hausärztlichen Versorgung}

Die Demenz vom Alzheimer Typ ist nach DSM-IV eine Kombinationsdiagnose aus

- beobachteten kognitiven Defiziten

- einem schleichenden, irreversiblen Verlauf und

- dem Ausschluss substanzinduzierter und systemischer Erkrankungen, ZNS-Erkrankungen bzw. eines Delirs.

Entsprechend gehört zur Diagnosestellung das Erheben einer Anamnese, eine körperliche Untersuchung, kognitive Leistungstests, Laboruntersuchungen sowie even- tuell bildgebende Diagnostik des Gehirns. Diese Anforderungen an eine Diagnose entstammen der Leitlinie Demenz der Universität Witten/Herdecke (3). Was für die hausärztliche Praxis jedoch fehlt, ist eine detaillierte Definition der Kriterien, die zur Diagnosestellung der Demenz vom Alzheimer-Typ unbedingt erforderlich sind.

In einer englischen DemenzLeitlinie (5) wird „zur Verbesserung des klinischen Urteils" des Hausarztes der Einsatz des MMSE oder des Uhrzeichentests empfohlen (ebenso in 19). Welchen Wert die Testergebnisse bei der Etablierung der Diagnose genau haben, bleibt allerdings offen. Welchen Wert für die Diagnosestellung hat z.B. ein unauffälliger MMSE, wenn der Patient klinisch dennoch kognitive Defizite zeigt?

Rösler et al. (12) beschreiben in ihrer Übersichtsarbeit mehr als 50 ältere und neuere standardisierte Untersuchungsinstrumente, die zur Erfassung der Demenz entwickelt wurden. Für die Routineversorgung in Klinik und Praxis werden die Blessed-, die GBS-Skala oder der SIDAM empfohlen. Die veranschlagte Untersuchungsdauer von mindestens 30 min relativiert die Einsetzbarkeit dieser Instrumente in der Praxisroutine jedoch erheblich.

Da also ein Standard in Bezug auf die Diagnosestellung der Demenz in der hausärztlichen Versorgung fehlt, ist es schwierig, Versorgungsdefizite festzumachen. Fehlt ein Goldstandard, ist eine behauptete Unterversorgung nicht präzise verifizierbar. So zeigte sich bei einer postalischen Befragung aller dänischen Hausärzte (18), dass $44 \%$ der Hausärzte selten oder nie bei Verdacht auf eine Demenz einen „Gedächtnistest“ zur Unterstützung ihrer Diagnostik heranzogen. Provozierend kann man da fragen: Ist das ein Defizit?

Nicht empfohlen wird in der Literatur, alle Patienten ab einem bestimmten Alter einem Screening auf kognitive Störungen zu unterziehen (1). Grund dafür ist die relativ niedrige Prävalenz der Demenz auch unter den älteren Patienten einer Hausarztpraxis, die in positiven prädiktiven Werten der Screeninguntersuchungen von unter $50 \%$ resultiert.
Früherkennung ohne Folgen?

Vorschläge in Richtung früherer Diagnosestellung basieren auf der Annahme, dass den Patienten dann besser geholfen werden könnte. Sandholzer et al. (14) untersuchten die Auswirkungen eines in Allgemeinarztpraxen durchgeführten Screenings auf kognitive Defizite. Mittels eines modifizierten MMSE wurden in 67 Allgemeinarztpraxen aus dem Hannoverschen und Leipziger Raum bei 18 ,6\% der über 70 Jährigen kognitive Beeinträchtigungen aufgedeckt, die dem Arzt bisher nicht bekannt waren. Zur großen Überraschung der Untersucher zogen die Ärzte aber praktisch nie eine unmittelbare diagnostische oder therapeutische Konsequenz aus dem positiven Screening-Ergebnis. Iliffe et al. (8) kamen in einer englischen Studie zu ähnlichen Ergebnissen. Was sind die Gründe für das passive Verhalten der Hausärzte?

\section{Wann und wie handeln \\ Hausärzte?}

In einer qualitativen Befragung von 28 niederländischen Hausärzten wurde der Versuch unternommen, das Handeln von Hausärzten in Bezug auf die Diagnose Demenz besser zu verstehen (7). Die Hausärzte gaben an, dass Hindernisse auf verschiedenen Ebenen die Frühdiagnose der Demenz erschwerten:

- Scham des Arztes, den Patienten auf kognitive Defizite zu testen und das Wort Demenz zu benutzen

- Leugnung der Defizite und der Diagnose seitens des Patienten, oft verbunden mit seltenen Konsultationen. Wenn der Hausarzt konsultiert würde, dann häufig aus anderen Gründen.

- Diagnostische Schwierigkeiten in den frühen Stadien der Demenz, z.B. fluktuierende Verläufe, unklare diagnostische Kriterien

- Übersehen von kognitiven Defiziten aufgrund der häufigen Multimorbidität der Patienten

- Zeitliche Beschränkungen in der normalen Sprechstunde.

Wagner und Abholz (17) betonen ferner die Bedeutung der von 
Hausärzten angenommenen „Therapiekonsequenz" für ihr diagnostisches Handeln: „Das, was ohne Therapiekonsequenz - nach ihrer Sicht - ist, muss nicht entdeckt und den Patienten möglicherweise belastend in den Vordergrund gestellt werden." Demzufolge handeln Hausärzte erst ab einem fortgeschrittenen Stadium der Demenz, wenn die pflegerische Versorgung in den Vordergrund rückt und die Angehörigen entlastet werden müssen. $\mathrm{Zu}$ deren Handeln gehört auch, absehbaren Krisen vorzubeugen, d.h. z.B. die Angehörigen auf das Auftreten von Inkontinenz frühzeitig vorzubereiten. Selbstkritisch merkten die Hausärzte in der niederländischen Studie (7) jedoch an, dass die Koordination häuslicher Hilfen manchmal Probleme bereite: Im Kontakt mit den Angehörigen stünde in der Sprechstunde - oft aus Zeitmangel vorwiegend das Befinden des Patienten im Vordergrund und die Einschätzung des tatsächlichen Hilfsbedarfs der Angehörigen erfolge nur unzureichend.

\section{Medikamentöse \\ Therapieoptionen}

Eine wichtige Rolle in der aktuellen Diskussion um die Frühdiagnose der Demenz spielen medikamentöse Therapieoptionen. Übereinstimmend mit verschiedenen Leitlinien werden von der Arzneimittelkommission der deutschen Ärzteschaft (16) für die Therapie demenzieller Erkrankungen die Cholinesterasehemmer Donepezil und Rivastigmin empfohlen, der Einsatz von Ginkgo biloba ist umstritten.

In einer postalischen Befragung bewerteten 563 deutsche Hausärzte (11) die medikamentösen Therapieoptionen bei Demenz durchaus positiv: $80 \%$ der Befragten stimmten der Aussage zu, dass „eine Verzögerung des kognitiven Abbaus für eine bestimmte Zeit durch Medikamente möglich ist." Auch Ruof et al. (13) fanden in einer telefonischen Befragung von 100 Hausärzten eine positive Bewertung von Cholinesterasehemmern: Einem eigenen nahen Angehörigen mit Alzheimer Demenz hätten $64 \%$ der befragten Hausärzte ein Medikament dieser Klasse ver- schrieben. Auf der anderen Seite ergab die Analyse der reellen Verschreibungsdaten derselben Hausärzte, dass sie nur 9\% ihrer Patienten mit Alzheimer Demenz einen Cholinesterasehemmer verschrieben. Als Begründung für die niedrigen Verschreibungsraten sahen die Autoren budgetäre Grenzen und sprachen von einer „verdeckten Rationierung."

Das Argument der Rationierung erscheint im Licht der Verschreibungsdaten allerdings relativ: So verordnete im Jahre 2001 im Durchschnitt jeder Hausarzt Medikamente in einem Umfang von ca. $239.000 €$ (10), was mehr ist, als der durchschnittliche Jahres-Bruttoumsatz einer Allgemeinarztpraxis. Dass die Cholinesterasehemmer im Jahre 2001 an allen verordneten Tagesdosen von Antidementiva nur einen Anteil von rund $4 \%$ erreichten, während Ginkgo-Präparate auf 56\% kamen (15), spricht dafür, dass die Hausärzte von der Wirksamkeit dieser Medikamente - zumindest in Relation zu deren Kosten - nicht überzeugt sind.

\section{- Die Kritik der Angehörigen}

In einer australischen Studie (2) gaben $89 \%$ der Angehörigen Demenzkranker an, mindestens einmal und mehrheitlich mehrfach einen Hausarzt wegen der Demenz konsultiert zu haben. Im Vergleich zu anderen Professionen wie Sozialarbeitern, Krankenschwestern oder ärztlichen Spezialisten wurden die Hausärzte als hilfreicher eingestuft. Dennoch kritisierten die Angehörigen das hausärztliche Verhalten als unzureichend: Während 43\% mit dem Hausarzt über die Diagnose gesprochen hatten, war nur bei $19 \%$ über die Prognose und nur bei 15\% über das Management der Demenz gesprochen worden.

Als Konsequenz schlugen die Autoren (2) vor, Hausärzte in Kommunikation zu schulen und in Fortbildungen ein stärkeres Gewicht auf die Problematik der Angehörigen zu legen.

Riedel-Heller et al. (11) weisen in ihrer Leipziger Untersuchung darauf hin, dass Hausärzte vorhandene Kooperationsmöglichkeiten zu wenig wahrnehmen. Nur knapp ein Drittel der Hausärzte hatte nach eigenen Angaben Kontakt zu Angehörigengruppen und nur 4\% zur „Alzheimergesellschaft“ oder zur „Hirnliga“. Offensichtlich sind Hausärzte über diese flankierenden Angebote, die sowohl den Angehörigen helfen als auch sie selbst entlasten, zu wenig informiert.

\section{Fazit}

Über die hausärztliche Versorgung von Patienten mit kognitiven Störungen und Demenz liegen nur wenige Studien vor, die zudem nicht immer Repräsentativität bzw. methodische Unanfechtbarkeit beanspruchen können. Zusammenfassend festzustellen ist, dass es bis heute an einem umfassenden Bild des diagnostischen und therapeutischen Geschehens in der primärärztlichen Versorgung in Deutschland fehlt. Ziel des Teilprojekts Hausärztliche Versorgung des Kompetenznetzes Demenzen ist es, einen ersten Schritt zur Beschreibung der Versorgungsrealität in Deutschland und damit eine Basis für eine Verbesserung der Versorgung zu schaffen.

\section{Das hausärztliche Versor- gungsprojekt des Kompe- tenznetzes Demenzen}

Das Hausärztliche Versorgungsprojekt ist Bestandteil des Projekts „Epidemiologie, Genetik und hausärztliche Versorgung“ (Projekt E.3.), das aus folgenden Teilprojekten besteht:

- E.3.1: Früherkennung von Patienten mit Hirnleistungsstörungen und Demenz in der hausärztlichen Versorgung (Früherkennungsprojekt) [Leitung: W. Maier (Bonn), M. C. Angermeyer (Leipzig), H. van den Bussche (Hamburg), H.-H. Abholz (Düsseldorf)].

- E.3.2: Identifikation von genetischen Markern und Haplotypen (Genetik-Projekt) [Leitung: W. Maier (Bonn), H. Kretzschmar (München), R. Heun (Bonn)].

- E.3.3: Hausärztliche Versorgung von Patienten mit Hirnleistungsstörungen und Demenz (Versorgungsprojekt) [(Leitung: H.-H. 
Abholz (Düsseldorf), H. van den Bussche (Hamburg)].

Das Projekt E.3.3 wird in den Studienzentren Hamburg, Düsseldorf, München, Mannheim, Bonn und Leipzig durchgeführt. Zum Zweck der besseren Überschaubarkeit der Ziele und Methoden wurde das Versorgungsprojekt in vier Module unterteilt, welche nachfolgend beschrieben werden.

\section{Modul 1: \\ Hausärztliche Versorgung von Patienten mit Hirnleis- tungsstörungen und Demenz}

\section{Zielsetzung der Untersuchung}

Zielsetzung dieses Moduls ist die Exploration der komplexen Handlungsstrategien von Hausärzten, wenn sie bei ihren Patienten mit Demenz oder Leichter Kognitiver Beeinträchtigung konfrontiert werden: Wann und wie kommt der Verdacht auf das Vorliegen einer kognitiven Störung auf? Welche diagnostischen und therapeutischen Schritte werden unternommen - und warum bzw. warum nicht? Welche kommunikativen Strategien mit Patienten und Angehörigen wenden die
Hausärzte an? Wie gehen sie mit einer Überforderung der Angehörigen um? Wie stehen sie zu Spezialsprechstunden, Patienten- und Angehörigengruppen etc.?

\section{Modul 2: \\ Die Einschätzung der kognitiven Leistungsfähig- keit von Patienten}

Fragestellung 1a: Welche Übereinstimmung besteht zwischen dem Urteil des Hausarztes und dem eines geschulten Untersuchers?

Methodik: Hausarzt und Untersucher schätzen zum Zeitpunkt $\mathrm{T}_{0}$ (d.h. bei Einschluss des Patienten in die E 3.1-Studie) die kognitive Leistungsfähigkeit des Patienten unabhängig voneinander anhand der Global Deterioration Scale (GDS) ein. Diese Einschätzungen werden miteinander vergleichen.

Fragestellung 1b: Wie gut kön-
nen Hausärzte den realen Verlauf
der kognitiven Leistungsfähig-
keit ihrer Patienten über drei
Jahre vorhersagen?

Methodik: Zum Zeitpunkt $\mathrm{T}_{0}$ machen die Hausärzte bei allen in die E.3.1-Studie eingeschlossenen Patienten eine Prognose anhand der GDS. Zum Zeitpunkt $T_{2}$ (d.h. bei der 2. Follow-up-Untersuchung nach 3 Jahren) schätzen die Untersucher die aktuelle kognitive Leistungsfähigkeit des Patienten ein. Beide Einschätzungen werden miteinander verglichen.

\section{Untersuchungsorte:}

Die Einschätzungen für Modul 2 werden an allen sechs Studienzentren für alle in die Studie eingeschlossenen Patienten erhoben, d.h. für ca. 120 Hausärzte und 3600 Patienten.

\section{Modul 3: \\ Kenntnisse der Hausärzte über Hirnleistungsstörungen und Demenz}

Hintergrund und Fragestellung:

Hausärzten wird häufig vorgehalten, dass ihre Kenntnisse hinsichtlich diagnostischer und therapeutischer Strategien bei demenziellen Erkrankungen unzureichend sind. Die Wissenslücken werden als

\section{Tab. 1 Methodik zu Modul 1: Untersuchungsinstrumente und Vorgehen}

\begin{tabular}{|c|c|c|c|}
\hline & Methodik & Anzahl der Befragten & Rekrutierung \\
\hline A & $\begin{array}{l}\text { Einstündige } \\
\text { Leitfadeninterviews }\end{array}$ & $\begin{array}{l}20 \text { Hausärzte in Hamburg und } \\
20 \text { Hausärzte in Düsseldorf, die nicht } \\
\text { mit dem Kompetenznetz verbunden sind }\end{array}$ & $\begin{array}{l}\text { Zufallsauswahl unter denjenigen Ärzten, } \\
\text { die nicht am Früherkennungsprojekt } \\
\text { teilnehmen; konsekutiver Einschluss }\end{array}$ \\
\hline B & $\begin{array}{l}\text { Standardisierte postalische } \\
\text { Befragung (Pilotierung) }\end{array}$ & 10 Hausärzte in Hamburg & $\begin{array}{l}\text { Fünf in A interviewte Ärzte und } 5 \text { Ärzte, } \\
\text { die nicht am Früherkennungsprojekt } \\
\text { beteiligt sind und in A auch nicht } \\
\text { interviewt wurden }\end{array}$ \\
\hline C & $\begin{array}{l}\text { Standardisierte postalische } \\
\text { Befragung }\end{array}$ & $\begin{array}{l}120 \text { am Früherkennungsprojekt teil- } \\
\text { nehmende Hausärzte und } 120 \text { nicht am } \\
\text { Früherkennungsprojekt teilnehmende } \\
\text { Hausärzte }\end{array}$ & $\begin{array}{l}\text { Die } 120 \text { nicht am Früherkennungsprojekt } \\
\text { teilnehmenden Hausärzte werden per } \\
\text { Zufall aus den Telefonbüchern der sechs } \\
\text { Studienzentren ausgewählt und sind } \\
\text { nicht identisch mit den Ärzten in A und B }\end{array}$ \\
\hline
\end{tabular}

Tab. 2 Die Kenntnisse der Hausärzte (Modul 3) werden auf zwei Wege ermittelt:

Methodik

Anzahl der Befragten

Rekrutierung

A Beantwortung eines MultipleChoice-Tests mit 25 Items
Die 120 nicht am Früherkennungsprojekt teilnehmenden Hausärzte werden per Zufall aus den Telefonbüchern der sechs Studienzentren ausgewählt.

B Bearbeitung zweier

Fallvignetten 
ein wichtiger Faktor für den beklagten „therapeutischen Nihilismus“ der Hausärzte angesehen. Ziel dieses Moduls ist die Einschätzung des Wissens von Hausärzten über Hirnleistungsstörungen und Demenz.

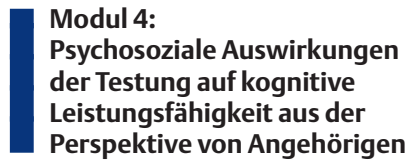

\section{Ausgangssituation und \\ Zielsetzung}

Bis heute gibt es keine Erkenntnisse über die psychologischen und sozialen Auswirkungen von Früherkennungsuntersuchungen auf Patienten und Angehörige. Es wird implizit angenommen, dass Testungen auf kognitive Defizite keine oder zumindest keine negativen Folgen für Patienten bzw. Angehörige haben, deren Realität somit nicht maßgeblich beeinflussen.

In diesem Untersuchungsmodul soll untersucht werden, ob dies tatsächlich der Fall ist. Wie erleben Patienten und ihre Angehörigen die Früherkennungsuntersuchungen? Was verändert sich dadurch? Welche Hoffnungen bzw. Enttäuschungen treten auf? Wie beurteilen die Angehörigen das Früherkennungsprojekt und das Kompetenznetz Demenzen?

\section{Methodik, Untersuchungsinstru- mente und Vorgehen}

Da zu diesem Themenbereich keine wissenschaftlichen Erkenntnisse vorhanden sind, wurde auch hier ein exploratives Vorgehen mit qualitativen Methoden gewählt. Dieses Vorgehen soll der Entwicklung und Begründung von Hypothesen für weitere Untersuchungen dienen. In Hamburg werden 20 halbstündige Leitfadeninterviews mit Angehörigen von im Früherkennungsprojekt E.3.1 getesteten Patienten durchgeführt.

Die Rekrutierung der Angehörigen erfolgt durch die Untersucher im Früherkennungsprojekt in der Weise, dass sie nach dem Interview die anwesenden Angehörigen fragen, ob sie generell Interesse an einem etwa halbstündigen Interview hätten. Die Auswahl der Angehörigen erfolgt nach inhaltlichen Gesichtspunkten, nicht nach solchen der Repräsentativität.

\section{Summary}

What is the reality of provision of health care to patients with cognitive impairment and dementia in general practice? This paper provides an overview about the available studies. Due to the fact that a standard for diagnosis of cognitive impairment and dementia in general practice is lacking, underdiagnosis is hard to demonstrate. On the other hand the literature provides an insight into constraints for early diagnosis. Against the background of the available literature this paper describes what the general practice project of the Competencenetwork Dementia will contribute to the clarification of problems in diagnosis and treatment in general practice.

\section{Key Words}

family practice - primary health care - general practice - dementia

\section{Literaturverzeichnis}

1. Brodaty H, Clarke J et al. Screening for Cognitive Impairment in General Practice: Toward a Consensus. Alzheimer Disease and Associated Disorders 1998; 12: 1-13

2. Brodaty H, Griffin D et al. A survey of dementia carers: doctors ' communications, problem behaviours and institutionals care. Australian and New Zealand Journal of Psychiatry 1990 ; 24 : 362-370

3. Butzlaff M, Koneczny $\mathrm{N}$ et al. Leitlinie Demenz. http://www.evidence.de/Leitlinien/ leitlinien.html

4. Cooper B, Bickel H et al. Demenzerkrankungen und leichtere kognitive Beeinträchtigungen bei älteren Patienten in der ärztlichen Allgemeinpraxis. Nervenarzt 1992; 63 : 551-560

5. Eccles M, Clarke J et al. North of England evidence based guidelines development project: guideline for the primary care management of dementia. BMJ 1998; 317 802-808

6. Eefsting JA, Boersma F et al. Differences in prevalence of dementia based on community survey and general practitioner recognition. Psychological Medicine1996; 26: 1223-1230

7. Van Hout H, Vernooij-Dassen M et al. General practitioners on dementia: tasks, practices and obstacles. Patient Education and Counseling 2000; 39: 219-225
8. Iliffe S, Mitchley S et al. Evaluation of the use of brief screening instruments for dementia, depression and problem drinking among elderly people in general practice. $\mathrm{Br}$ J Gen Pract 1994; 44: 503-507

9. Mant A, Eyland EA et al. Recognition of Dementia in General Practice: Comparison of General Practitioners' Opinions with Assessments Using the Mini-Mental State Examination and the Blessed Dementia Rating Scale. Family Practice 1988; 5: 184-188

10. Nink K, Schröder H. Arzneiverordnungen nach Arztgruppen. In: Schwabe U, Paffrath D (Hrsg.). Arzneiverordnungs-Report 2002. Berlin, Heidelberg, Springer-Verlag, 2003: 907-917

11. Riedel-Heller SG, Schork A et al. Demenzkranke in der Hausarztpraxis - Ergebnisse einer Befragung. Z Gerontol Geriat 2000; 33: 300-306

12. Rösler M, Frey U et al. Diagnostik der Demenzen: Standardisierte Untersuchungsinstrumente im Überblick. Fortschr Neurol Psychiat 2003; 71: 187-198

13. Ruof J, Mittendorf T et al. Diffusion of innovations: treatment of Alzheimer's disease in Germany. Health Policy 2002; 60: 59-66 14. Sandholzer H, Breull A et al. Früherkennung und Frühbehandlung von kognitiven Funktionseinbußen: eine Studie über eine geriatrische Vorsorgeuntersuchung im unausgelesenen Patientengut der Allgemeinpraxis. Z Gerontol Geriat 1999; 32: 172-178 15. Schwabe U. Antidementiva. In: Schwabe U, Paffrath D (Hrsg.). ArzneiverordnungsReport 2002. Berlin, Heidelberg, SpringerVerlag. 2003: 156-169

16. Therapieempfehlungen der Arzneimittelkommission der deutschen Ärzteschaft, Demenz. 2. Auflage 2001 (www.akdae.de) 17. Wagner G, Abholz HH. Diagnose und Therapiemanagement der Demenz in der Hausarztpraxis. Z Allg Med 2002; 78: 239-244

18. Waldorff FB, Moller S Diagnostic evaluation of dementia in general practice in Denmark. Scand J Prim Health Care 2001; 19: 117-121

19. Wind AW, Schellevis FG et al. Limitations of the Mini-Mental State Examination in diagnosing dementia in general practice. Int J Geriatr Psychiat 1997; 12: 101-108

\section{Korrespondenzadressen:}

Dr. med. Hanna Kaduszkiewicz Prof. Dr. med. Hendrik van den Bussche Institut für Allgemeinmedizin

Zentrum für Psychosoziale Medizin

Universitätsklinikum Hamburg-Eppendorf

Martinistr. 52

20246 Hamburg

Email: kaduszki@uke.uni-hamburg.de 